\title{
Vier Schlüsselfragen zur adjuvant-endokrinen Therapie
}

\author{
Gesprächsleitung: Peter Dalla \\ Teilnehmer: Jens Huober ${ }^{b} \quad$ Marcus Schmidt $^{c} \quad$ Florian Schütz $^{\mathrm{d}} \quad$ Michael Untch $^{\mathrm{e}}$ \\ ${ }^{a}$ Frauenklinik, Städtisches Klinikum Lüneburg, Germany \\ ${ }^{\mathrm{b}}$ Kantonsspital St. Gallen, Switzerland \\ ${ }^{\mathrm{c}}$ Klinik und Poliklinik für Geburtshilfe und Frauenheilkunde, Johannes Gutenberg-Universität, Mainz, \\ d Universitätsfrauenklinik Heidelberg, \\ ${ }^{e}$ Frauenklinik, HELIOS Klinikum Berlin-Buch, Germany
}

Frage 1: Bei welcher Patientin mit rezeptorpositivem Mammakarzinom würden Sie mit einem

Aromatasehemmer die endokrine Therapie beginnen?

Huober: Bei den Patientinnen mit einem hohen oder intermediären Risikoprofil würde ich aufgrund des Rezidivpeaks in den ersten 2 Jahren die endokrine Therapie mit einem Aromatasehemmer (AI) beginnen. Bei Patientinnen mit einem niedrigen Risikoprofil ist nach wie vor eine alleinige Tamoxifentherapie eine mögliche Option. Es ist jedoch zu beachten, dass je nach Tumorprofil die Unterschiede zwischen den verschiedenen Behandlungsstrategien klein sein können und die Verträglichkeit und die Beeinträchtigung des Gesundheitsstatus durch eine Behandlung und natürlich auch der Patientinnenwunsch wichtige Entscheidungskriterien sind. Bei prämenopausalen Patientinnen hat eine AI-Therapie außerhalb von Studien keinen Stellenwert.

Schmidt: Da ein Vorteil für das Gesamtüberleben bislang nur in Studien mit der Sequenz- oder Switchstrategie gezeigt werden konnte, ist aus meiner Sicht die Hauptindikation für den Beginn der adjuvanten endokrinen Therapie mit einem AI eine Kontraindikation gegen Tamoxifen.

Schütz: Gemäß den Daten der BIG-1-98-Studie profitieren besonders diejenigen Patientinnen mit nodal-positiver Erkrankung in den ersten zwei Jahren einer Therapie mit einem AI. In früheren Analysen scheinen auch diejenigen Patientinnen mit schnell proliferierenden Tumoren und einem hohen Ki67 (>20\%) einen höheren Benefit vom AI zu haben. Bei diesen beiden Patientengruppen beginne ich die Therapie mit einem AI.
Untch: Die 100-Monats-Analyse der ATAC-Studie zum Vergleich von Anastrozol mit Tamoxifen bei hormonrezeptorpositiven postmenopausalen Mammakarzinompatientinnen, publiziert im Lancet 2008, zeigt keinerlei Benefit von Anastrozol verglichen mit Tamoxifen in Bezug auf das Gesamtüberleben. Aus diesem Grunde wurde in der AGO-Leitliniengruppe 2010 die Empfehlung AI upfront mit einem einfachen + versehen.

Die Daten zum Erstlinieneinsatz von Letrozol im Vergleich zu Tamoxifen aus der BIG-1-98-Studie, die aktuell von Mouridsen im New England Journal of Medicine publiziert wurden, zeigen in der Intention-to-treat-Analyse im letzten Follow-up ebenfalls keinen Überlebensvorteil. Da nach Bekanntgabe der ersten Rezidivdaten aus dieser Studie ein nicht unwesentlicher Teil der Patientinnen aus dem TamoxifenKontrollarm anschließend Letrozol bekamen, ergab eine zensierte Analyse einen signifikanten Vorteil für das Überleben. Dies ist jedoch aus statistischen Gründen keine saubere Analyse, sodass die Wahrheit wahrscheinlich irgendwo in der Mitte zwischen Intention-to-treat- und zensierter Analyse liegt.

Bei den meisten Patientinnen, die in unserem Brustzentrum operiert werden, wird eine sogenannte Sequenztherapie durchgeführt: 2-3 Jahre Tamoxifen, gefolgt von 2-3 Jahren AI. Für diese Sequenztherapie gibt es das höchste Evidenzlevel aus mehreren Studien in Bezug auf einen Überlebensvorteil verglichen mit einer alleinigen Tamoxifentherapie.

Um Ihre Anfangsfrage zu beantworten: Patientinnen mit einem sehr hohen Metastasierungsrisiko, z.B. hochgradiger Lymphknotenbefall, großer Tumor, schlechtes Grading, sind Kandidatinnen für eine Upfront-AI-Therapie. Ebenso auch Patientinnen, bei denen sich der Einsatz von Tamoxifen verbietet, z.B. bei venöser Thromboembolie-Anamnese.

\section{KARGER}

Fax +497614520714

Information@Karger.de

www.karger.com (c) 2010 S. Karger GmbH, Freiburg

Accessible online at:

www.karger.com/brc 
Frage 2: Gibt es noch einen Stellenwert für die alleinige fünfjährige Al-Therapie oder kann in jedem Fall Tamoxifen mit eingeschlossen werden?

Huober: Die alleinige AI-Therapie hat für mich nach wie vor einen Stellenwert in der adjuvanten endokrinen Behandlung. Die Daten der BIG 1-98 lassen wohl den Schluss zu, dass nach einer 2-jährigen AI-Therapie auf Tamoxifen gewechselt werden kann, da die bisherigen Analysen keine Unterschiede dieser Sequenz (Letrozol-Tamoxifen) in der Anzahl der der brustkrebsbedingten Ereignisse im Vergleich zur alleinigen AI-Therapie zeigten. Vor einer abschließenden Bewertung sollte aber eine längere Nachbeobachtung abgewartet werden. Eine retrospektive Auswertung dieser Studie von G. Viale zeigt, dass zumindest bei Patientinnen mit High-riskMerkmalen eine 5-jährige AI-Therapie eventuell doch den Sequenzen überlegen ist. Wenn eine Patientin die AI-Therapie gut verträgt ergibt sich für mich daher keine Indikation nach 2 Jahren auf Tamoxifen zu wechseln. Tamoxifen ist auch nicht notwendigerweise für jede Patientin die Substanz mit dem günstigeren Nebenwirkunsprofil.

Schmidt: Wie oben angeführt, sehe ich den Stellenwert für eine alleinige AI-Therapie vor allem bei Tamoxifenkontraindikationen. Tamoxifen sollte, wenn möglich, in der adjuvanten endokrinen Therapie weiterhin berücksichtigt werden.

Schütz: Tamoxifen kann gemäß den Daten der BIG-1-98-Studie bezüglich der Effektivität immer in das Therapiekonzept im Sinne einer inversen Sequenz (AI-Tamoxifen) eingebunden werden. Frauen mit starken klimakterischen Beschwerden in der Anamnese leiden jedoch häufiger unter einer Therapie mit Tamoxifen, so dass ich bei diesen Patientinnen eher eine 5-jährige AI-Therapie favorisieren würde.

Untch: In meinen Augen ist eine alleinige 5-jährige Aromatasehemmer-Therapie bei denjenigen Patientinnen angesagt, die nach 2-3 Jahren AI-Therapie rezidiv- und metastasenfrei sind und keine wesentlichen Nebenwirkungen von dieser Therapie haben. Ansonsten kann grundsätzlich jede Patientin nach 2-3 Jahren AI-Therapie wieder umgestellt werden auf Tamoxifen, da diese sogenannte reverse Sequenz in der BIG1-98-Studie keinen Nachteil gegenüber 5 Jahren durchgehender AI-Therapie gezeigt hat und die Verträglichkeit im Allgemeinen besser ist.

\section{Frage 3: Für welche Patientinnen würden Sie eine länger als 5 Jahre andauernde endokrine Therapie für sinnvoll halten?}

Huober: Primär für die postmenopausale Patientin mit initial nodal positiver Erkrankung, da bei diesen Patientinnen auch nach 5 rezidivfreien Jahren in den darauf folgenden 5 Jahren die Rezdidivrate doppelt so groß wie bei nodal negativer Krankheit ist. Außerdem haben diese Patientinnen in der MA17-Studie von einer erweiterten adjuvanten Therapie auch mit einem verbesserten Gesamtüberleben profitiert. In diesem Zusammenhang möchte ich auf die SOLE-Studie hinweisen, die auch in Deutschland offen ist, wobei hier bei nodal positiven postmenopausalen Patientinnen nach 5 Jahren endokriner Therapie eine kontinuierliche Behandlung mit Letrozol mit einer intermittiernden Letrozolbehandlung (9 Monate Therapie, 3 Monate Pause) über jeweils 5 Jahre überprüft wird. Eine Unterbrechung der Östradiolsuppression kann eventuell eine endokrine Resistenzbildung verhindern.

Schmidt: Eine genauere Berücksichtigung der Tumorbiologie zeigt, dass die überwiegende Mehrzahl der frühen Brustkrebsereignisse (zumeist Fernmetastasen) vor allem bei basal-likedifferenzierten oder HER2-positiven Mammakarzinomen auftreten. Die Langzeitbeobachtung dieser Patientinnen zeigt weiterhin, dass speziell bei den basal-like-Mammakarzinomen die Metastasierungshäufigkeit nach 3-5 Jahren stark abnimmt. Demgegenüber können luminal differenzierte Mammakarzinome, die positiv für den Östrogenrezeptor (ER) sind, Metastasen oft auch nach mehr als 5 Jahren ausbilden.

Aus diesem Grund wäre es gerade bei ER-positiven Patientinnen sinnvoll, die adjuvante Therapie länger als 5 Jahre durchzuführen. Auf der anderen Seite muss natürlich das grundsätzliche Risiko, ob überhaupt Metastasen auftreten, berücksichtigt werden, da eine lang andauernde adjuvante endokrine Therapie potentiell auch Nebenwirkungen haben kann. Derzeit ist speziell das Risiko des Auftretens von Metastasen nach 5 Jahren durch tumorbiologische Untersuchungen nur schwer abzubilden, so dass es gerechtfertigt scheint, sich an klassischen Prognosefaktoren wie dem Nodalstatus zu orientieren. In prospektiven Studien konnte gezeigt werden, dass speziell nodal positive Patientinnen von einer länger als 5 Jahre andauernden adjuvanten endokrinen Therapie profitieren können.

Schütz: Nodal positive Patientinnen, welche eine 5-jährige Tamoxifentherapie erhalten hatten und am Ende dieser 5 Jahre sicher postmenopausal sind (gegebenenfalls Hormonstatus nach Absetzen von Tamoxifen) sollten über weitere 5 Jahre mit einem AI behandelt werden. Für andere Indikationen gibt es momentan noch keine Evidenz. Jedoch sollten wir uns meiner Meinung nach auf eher längere endokrine Therapieintervalle einstellen.

Untch: Eine 5-jährige endokrine Therapie ist bislang als Standard anzusehen, meist bestehend aus der Sequenz Tamoxifen gefolgt von einem AI. Patientinnen mit Hormonrezeptorpositivem Mammakarzinom haben ein niedriges, jedoch über lange Jahre andauerndes Rezidiv- und Metastasenrisiko. Leider haben alle bisher durchgeführten Studien bis auf wenige Ausnahme eine länger dauernde Therapie als 5 Jahre nicht 
getestet. Insofern kann man hier keine generelle Empfehlung für eine länger als 5 Jahre dauernde Therapie geben. Die einzige Studie, die einen Vorteil einer länger dauernden Therapie gezeigt hat, war die Studie von Paul Goss, in der nach 5 Jahren Tamoxifen Letrozol verglichen mit Placebo eingesetzt wurde. Für den Einsatz von Letrozol wurde ein signifikanter Überlebensvorteil bei Patientinnen mit befallenen axillären Lymphknoten gezeigt. Mit anderen Worten: Wenn eine Patientin nach 5 Jahren Tamoxifen sich in der Klinik oder Praxis mit dem Wunsch einer weiteren Therapie vorstellt, ist die erweiterte adjuvante Therapie eine sinnvolle Option, siehe auch AGO-Leitlinie 2010.

\section{Frage 4: Inwieweit können Genexpressionsanalysen wie z.B. der Onkotype DX-Assay oder Enzym-Analysen wie bei CYP2D6 bei der adjuvant endokrinen Therapieentscheidung helfen? Wie sehen Sie im Falle eines Nutzens dieser Verfahren gegebenenfalls den zeitlichen Rahmen bis zur Standardisierung und deutschlandweiten Anwendung?}

Huober: Beide Verfahren spielen momentan für die Therapieentscheidung im klinischen Alltag keine Rolle, da die Datenlage nicht ausreichend bzw. im Falle von CYP2D6 auch widersprüchlich ist. Einer Patientin bei dieser unsicheren Datenlage (und noch nicht eindeutig validierten Testverfahren) Tamoxifen wegen einer CYP2D6-Testung vorzuenthalten halte ich für sehr problematisch und kann davon nur abraten. Ich glaube nicht, dass wir in naher Zukunft mit einer generellen Empfehlung rechnen können.

Schmidt: Auch wenn wir uns derzeit, wie oben angesprochen, vor allem an klassischen Prognosefaktoren orientieren, besteht eindeutig die Notwendigkeit einer besseren tumorbiologisch orientierten Prognoseabschätzung. MikroarrayAnalysen, die tausende Gene in ihrer Expression simultan messen, haben zu neuen Einsichten in die Tumorbiologie des Mammakarzinoms geführt und gezeigt, dass neben dem Östrogenrezeptor und der Proliferation auch das tumorassoziierte Immunsystem eine wichtige prognostische Rolle spielt. Mikroarrayanalysen haben allerdings den Nachteil, dass bei $-80{ }^{\circ} \mathrm{C}$ frisch eingefrorenes Tumorgewebe vorliegen muss. Aus Gründen der Praktikabilität sind daher Analysen mittels RT-PCR, die am Paraffinmaterial durchgeführt werden können, zu bevorzugen. Diesen Ansatz verfolgen Tests wie Oncotype $\mathrm{DX}^{\circledR}$, der mehrere bekannte und gut untersuchte Marker wie zum Beispiel ER, HER2 oder den Proliferationsmarker Ki-67 enthält. Ein darüber hinaus gehender und möglicherweise sinnvollerer Ansatz besteht darin, die Erkenntnisse, die zunächst durch mikroarraybasierte Genexpressionsanalysen gewonnen wurden, mit wenigen $(\sim 10)$ Genen, die dann im Paraffinmaterial untersucht werden können, umzusetzen.
Entscheidend bei einem prognostischen oder prädiktiven Testverfahren ist, dass es an einem unabhängigen Kollektiv validiert wird. Zur Validierung sollte bevorzugt Paraffinmaterial aus einer klinischen Studie verwendet werden, da hier ein nach definierten Kriterien ausgewähltes und homogen behandeltes Patientinnenkollektiv vorliegt. Standardisierung und Qualitätskontrolle lassen sich bei RT-PCR basierten Tests gut erreichen. Der potentielle Nachteil einer mangelnden morphologischen Kontrolle (ist das, was gemessen wird, wirklich der Tumor?) solcher molekularbiologischer Testverfahren gegenüber der herkömmlichen Immunhistochemie kann durch Auswahl eines repräsentativen Tumorareals am Paraffinschnitt zuverlässig vermieden werden. Ein Vorteil liegt sicherlich darin, dass Untersucherabhängigkeit keine Rolle spielt. Aus meiner Sicht haben RT-PCR-basierte diagnostische Testverfahren eine realistische Chance, innerhalb der nächsten 2 Jahre in der Routinediagnostik eine wichtige Rolle für die prognostische Einschätzung beim Mammakarzinom zu spielen.

Schütz: Genexpressionsanalysen wie Oncotype DX können zukünftig eventuell helfen, eine Indikation für oder gegen eine adjuvante Chemotherapie zu stellen. Die Indikation, ob eine endokrine Therapie überhaupt notwendig sein mag, ist momentan nicht Gegenstand der Forschung. Ein routinemäBiger Einsatz kann (noch) nicht empfohlen werden, auch wenn retrospektive Analysen erfolgversprechend sind.

Enzym-Analysen hingegen sollen die Entscheidung erleichtern, welche endokrine Therapie abhängig von der individuellen Situation der Patientin zu wählen ist. Für letztere Untersuchungen gibt es divergierende Ergebnisse, so dass eine Empfehlung diesbezüglich noch nicht abzugeben ist.

Untch: Der Oncotype DX-Assay wie auch die Untersuchung mit dem MammaPrint-Test wurden in großen retrospektiven Analysen validiert. Beide Testsysteme werden zur Zeit auch in großen internationalen Studien prospektiv überprüft (TailorX-Studie in USA, Mindact-Studie in Europa, Plan BStudie in Deutschland usw.). Der einzige Test, der nicht nur retrospektiv, sondern auch prospektiv im Rahmen von Therapiestudien überprüft wurde, ist die Messung der Proteasen uPA/PAI1 im frischen Tumorgewebe. Hierzu muss zwar eine gewisse Logistik in der Pathologie vorhanden sein, jedoch ist die Testung routinemäßig machbar und sehr kostengünstig. Dieses Testsystem erlaubt es, gerade bei nodal negativen Patientinnen mit hormonrezeptorpostivem Status und G2 in etwa $40 \%$ der Fälle eine Chemotherapie zu vermeiden, nämlich bei Patientinnen, die nicht erhöhte Proteasen haben. Hingegen hat der Einsatz der adjuvanten Chemotherapie bei dieser Patientinnengruppe mit erhöhten Proteasen einen signifikanten Vorteil in Bezug auf krankheitsfreies und Gesamtüberleben gezeigt.

Bei der CYP2D6-Testung gibt es ebenfalls interessante retrospektive Daten: möglicherweise haben sogenannte «Nied- 
rigmetabolisierer» von Tamoxifen keinerlei Benefit. $\mathrm{Ob}$ genau diese Patientinnen Kandidatinnen für einen AI sind, konnten die Studien bisher nicht beweisen. Dazu müsste man prospektive Studien durchführen. Aus diesem Grunde kann für eine routinemäßige CYP2D6-Testung keine generelle Empfehlung gegeben werden. Im Übrigen sind sowohl für den uPA/PAI1-Test wie auch für den CYP2D6-Test diese Empfehlungen in die Leitlinien der AGO 2010 eingegangen.

\section{Teilnehmer:}

Prof. Dr. Jens Huober Kantonsspital St. Gallen 9007 St. Gallen, Switzerland Jens.huober@kssg.ch

Dr. med. Marcus Schmidt

Klinik und Poliklinik für Geburtshilfe und Frauenheilkunde Johannes Gutenberg-Universität

Langenbeckstr. 1, 55131 Mainz, Germany

marcus.schmidt@frauen.klinik.uni-mainz.de

Dr. med. Florian Schütz

Universitätsfrauenklinik Heidelberg

Zeppelinstr. 11-33, 69121 Heidelberg, Germany

florian_schuetz@med.uni-heidelberg.de

Prof. Dr. med. Michael Untch

Frauenklinik

HELIOS Klinikum Berlin-Buch

Schwanebecker Chaussee 50, 13125 Berlin, Germany

michael.untch@helios-kliniken.de 